Bull. Soc. math. France

130 (4), 2002, p. $603-618$

\title{
SYMMETRIES OF THE NONLINEAR SCHRÖDINGER EQUATION
}

\author{
By Benoît Grébert \& Thomas Kappeler
}

\begin{abstract}
Symmetries of the defocusing nonlinear Schrödinger equation are expressed in action-angle coordinates and characterized in terms of the periodic and Dirichlet spectrum of the associated Zakharov-Shabat system. Application: proof of the conjecture that the periodic spectrum $\cdots<\lambda_{k}^{-} \leq \lambda_{k}^{+}<\lambda_{k+1}^{-} \leq \cdots$ of a ZakharovShabat operator is symmetric, i.e. $\lambda_{k}^{ \pm}=-\lambda_{-k}^{\mp}$ for all $k$, if and only if the sequence $\left(\gamma_{k}\right)_{k \in \mathbb{Z}}$ of gap lengths, $\gamma_{k}:=\lambda_{k}^{+}-\lambda_{k}^{-}$, is symmetric with respect to $k=0$.

RÉSumÉ (Symétries de l'équation de Schrödinger non linéaire). - Les symétries de l'équation de Schrödinger nonlinéaire sont exprimées dans les variables action-angles et caractérisées à l'aide du spectre périodique et du spectre de Dirichlet du système de Zakharov-Shabat associé. Comme application, nous démontrons la conjecture suivante : le spectre périodique $\cdots<\lambda_{k}^{-} \leq \lambda_{k}^{+}<\lambda_{k+1}^{-} \leq \cdots$ de l'opérateur de ZakharovShabat est symétrique, i.e. $\lambda_{k}^{ \pm}=-\lambda_{-k}^{\mp}$ pour tout $k$, si et seulement si la suite $\left(\gamma_{k}\right)_{k \in \mathbb{Z}}$ des longueurs des intervalles d'instabilité, $\gamma_{k}:=\lambda_{k}^{+}-\lambda_{k}^{-}$, est symétrique par rapport à $k=0$.
\end{abstract}

Texte reçu le 8 octobre 2001, accepté le 24 mai 2002

Benoît GréBert, Département de Mathématiques, UMR 6629 CNRS, Université de Nantes, 2, rue de la Houssinière, BP 92208, 44322 Nantes Cedex 03 (France)

E-mail : grebert@math.univ-nantes.fr • Url : http://www.math.sciences.univnantes.fr/ grebert/

Thomas Kappeler, Institut für Mathematik, Universität Zürich, Winterthurerstrasse 190, CH-8057 Zürich (Switzerland) • E-mail : tk@math.unizh.ch Url : www.math.unizh.ch/kappeler/

2000 Mathematics Subject Classification. - 35Q55, 37K10, 37L20, 34A55.

Key words and phrases. - NLS equation, Zakharov-Shabat operators, action-angle variables, symmetries. 


\section{Introduction}

The defocusing nonlinear Schrödinger equation NLS on the circle

$$
i \partial_{t} \varphi=-\partial_{x}^{2} \varphi+2|\varphi|^{2} \varphi
$$

can be viewed as a completely integrable Hamiltonian system of infinite dimension. Indeed, on the phase $L^{2}\left(S^{1} ; \mathbb{C}\right)$, introduce the Poisson bracket

$$
\{F, G\}:=i \int_{S^{1}}\left(\frac{\partial F}{\partial \varphi(x)} \cdot \frac{\partial G}{\partial \bar{\varphi}(x)}-\frac{\partial F}{\partial \bar{\varphi}(x)} \cdot \frac{\partial G}{\partial \varphi(x)}\right) \mathrm{d} x .
$$

Equation (1.1) can then be written in Hamiltonian form as follows

$$
\frac{\partial \varphi}{\partial t}=\{\mathcal{H}, \varphi\}=-i \frac{\partial \mathcal{H}}{\partial \bar{\varphi}}, \quad \frac{\partial \bar{\varphi}}{\partial t}=\{\mathcal{H}, \bar{\varphi}\}=i \frac{\partial \mathcal{H}}{\partial \varphi},
$$

where the Hamiltonian $\mathcal{H}$ is given by $(c f .[2])$

$$
\mathcal{H}(\varphi):=\int_{S^{1}}\left(\left|\frac{\partial \varphi}{\partial x}\right|^{2}+|\varphi|^{4}\right) \mathrm{d} x .
$$

Consider the following symmetry operators, acting on $L^{2}\left(S^{1} ; \mathbb{C}\right)$,

$$
\begin{gathered}
\mathcal{S}_{1}(\varphi):=\bar{\varphi}, \quad \mathcal{S}_{2}(\varphi)=\check{\varphi}, \\
M_{\alpha} \varphi:=\mathrm{e}^{i \alpha} \varphi, \quad T_{\tau} \varphi:=\varphi(\tau+\cdot),
\end{gathered}
$$

where $\check{\varphi}$ is defined by $\check{\varphi}(x)=\varphi(-x)$. For convenience, we introduce $\mathcal{S}_{3}:=M_{\pi}$, i.e. $\mathcal{S}_{3}(\varphi)=-\varphi$. Notice that the Hamiltonian $\mathcal{H}$ is invariant under $\mathcal{S}_{1}, \mathcal{S}_{2}, M_{\alpha}$ and $T_{\tau}$.

Denote by $U(t)$ the solution operator of $(1.1)$ for initial data in $L^{2}\left(S^{1} ; \mathbb{C}\right.$ ) (or some Sobolev space $\left.H^{N}\left(S^{1} ; \mathbb{C}\right)\right)$ (cf [1]). It is immediate that $U(t)$ commutes with $\mathcal{S}_{2}, \mathcal{S}_{3}, M_{\alpha}$ and $T_{\tau}$ and that

$$
U(t) \mathcal{S}_{1}=\mathcal{S}_{1} U(-t)
$$

Recall that NLS admits a Lax pair representation

$$
\frac{\mathrm{d} L}{\mathrm{~d} t}=[L, A]
$$

where $L=L(\varphi)$ is the Zakharov-Shabat operator

$$
L(\varphi):=i\left(\begin{array}{rr}
1 & 0 \\
0 & -1
\end{array}\right) \frac{\mathrm{d}}{\mathrm{d} x}+\left(\begin{array}{ll}
0 & \varphi \\
\bar{\varphi} & 0
\end{array}\right)
$$

and $A$ is a (rather complicated) operator given in [2]. We remark that $L(\varphi)$ is unitarily equivalent to the well known AKNS-operator

$$
H(\varphi):=\left(\begin{array}{rr}
0 & -1 \\
1 & 0
\end{array}\right) \frac{\mathrm{d}}{\mathrm{d} x}+\left(\begin{array}{rr}
-q & p \\
p & q
\end{array}\right)
$$

where $\varphi=-q+i p$, a fact which will be used throughout the paper.

TOME $130-2002-\mathrm{N}^{\mathrm{O}} 4$ 
Denote by $\operatorname{spec}_{\text {per }} L(\varphi)$ the periodic spectrum of $L(\varphi)$ when considered on the interval $[0,2]$ and by $\operatorname{spec}_{\text {Dir }}^{ \pm} L(\varphi)$ the Dirichlet spectra of $L(\varphi)$ when considered on the interval $[0,1]$ (cf. Definitions (2.5) and (2.6) below). The operator $L(\varphi)$ is selfadjoint when considered with periodic or Dirichlet boundary conditions. Hence both $\operatorname{spec}_{\text {per }} L(\varphi)$ and $\operatorname{spec}_{\text {Dir }} L(\varphi)$ are real.

By elementary considerations one shows that

$$
\begin{aligned}
& \operatorname{spec}_{\text {per }} L(\bar{\varphi})=-\operatorname{spec}_{\text {per }} L(\varphi), \quad \operatorname{spec}_{\text {per }} L(\check{\varphi})=-\operatorname{spec}_{\text {per }} L(\varphi), \\
& \operatorname{spec}_{\text {per }} L\left(M_{\alpha} \varphi\right)=\operatorname{spec}_{\text {per }} L(\varphi), \quad \operatorname{spec}_{\text {per }} L\left(T_{\tau} \varphi\right)=\operatorname{spec}_{\text {per }} L(\varphi)
\end{aligned}
$$

and expresses $\operatorname{spec}_{\text {Dir }}^{+} L\left(\mathcal{S}_{j} \varphi\right)$ for $j=1,2,3$ in terms of $\operatorname{spec}_{\text {Dir }}^{-} L(\varphi)$.

Recall from [7] (see also [8]) that NLS admits global Birkhoff coordinates. Denote by $\ell^{2}\left(\mathbb{Z} ; \mathbb{R}^{2}\right)$ the space of $\ell^{2}$-sequences $\left(x_{j}, y_{j}\right)_{j \in \mathbb{Z}}$ endowed with the canonical Poisson bracket $\left\{x_{i}, x_{j}\right\}=0,\left\{y_{i}, y_{j}\right\}=0$ and $\left\{x_{i}, y_{j}\right\}=\delta_{i j}$.

THEOREM 1.1. - There exists a canonical diffeomorphism $\Phi$

$$
\Phi: \ell^{2}\left(\mathbb{Z} ; \mathbb{R}^{2}\right) \longrightarrow L^{2}\left(S^{1} ; \mathbb{C}\right)
$$

such that

1) $\Phi$ is bianalytic;

2) the restriction of $\Phi$ to the weighted $\ell^{2}$-space $\ell_{N}^{2}\left(\mathbb{Z} ; \mathbb{R}^{2}\right)(N \geq 1)$ is a diffeomorphism onto the Sobolev space $H^{N}\left(S^{1} ; \mathbb{C}\right)$;

3) $\left(x_{j}, y_{j}\right)_{j \in \mathbb{Z}}=\Phi^{-1}(\phi)$ are Birkhoff coordinates for NLS and its hierarchy, i.e. any Hamiltonian in the hierarchy is a function of the actions $I_{j}:=\frac{1}{2}\left(x_{j}^{2}+\right.$ $\left.y_{j}^{2}\right)$ only.

In this article we use the explicit formulas for action and angle variables given in [8] (see also [7]) to obtain

TheOREM 1.2. - (i) The actions are left invariant by $M_{\alpha}$ and $T_{\tau}$, i.e. for any $k \in \mathbb{Z}$

$$
I_{k}\left(M_{\alpha} \varphi\right)=I_{k}(\varphi) \quad \text { and } \quad I_{k}\left(T_{\tau} \varphi\right)=I_{k}(\varphi)
$$

whereas $I_{k}(\bar{\varphi})$ and $I_{k}(\breve{\varphi})$ can be computed to be $(j=1,2)$

$$
I_{k}\left(\mathcal{S}_{j} \varphi\right)=I_{-k}(\varphi) .
$$

(ii) For $k$ with $I_{k} \neq 0$

$$
\begin{aligned}
\theta_{k}\left(M_{\alpha} \varphi\right) & \equiv \theta_{k}+\alpha \quad(\bmod 2 \pi), \\
\theta_{k}(\breve{\varphi}) & \equiv \theta_{-k}(\varphi) \quad(\bmod 2 \pi), \\
\theta_{k}(\bar{\varphi}) & \equiv-\theta_{-k}(\varphi) \quad(\bmod 2 \pi) .
\end{aligned}
$$

As a first application of Theorem 1.2 one obtains ( $c f$. Proposition 4.1 in Section 4 )

BULLETIN DE LA SOCIÉtÉ MATHÉMATIQUE DE FRANCE 
Corollary 1.1. - When evaluated at $I=\left(I_{k}\right)_{k \in \mathbb{Z}}$ with $I_{k}=I_{-k}$ for all $k \in \mathbb{Z}$, the $N L S$ frequencies $\omega=\left(\omega_{k}\right)_{k \in Z}, \omega_{k}=\partial \mathcal{H} / \partial I_{k}$, are symmetric, i.e. $\omega_{k}(I)=\omega_{-k}(I)$ for all $k \in \mathbb{Z}$.

The main motivation for proving Theorem 1.2 and Corollary 1.1 comes from an application to a KAM type theorem established in [5] (see also [6]).

As a second application, Theorem 1.2 is used to prove that the periodic spectrum is symmetric if and only if the sequence of the gap lengths is symmetric, a conjecture, raised by several experts in the field. More precisely, denote by

$$
\operatorname{spec}_{\text {per }} L(\varphi)=\left(\lambda_{k}^{ \pm}(\varphi)\right)_{k \in \mathbb{Z}}
$$

the periodic spectrum of $L(\varphi)$ when considered on the inverval [0,2] where the numbers $\lambda_{k}^{ \pm}(\varphi)$ are ordered so that

$$
\lambda_{k}^{-}(\varphi) \leq \lambda_{k}^{+}(\varphi)<\lambda_{k+1}^{-}(\varphi)
$$

and let $\gamma(\varphi):=\left(\gamma_{k}(\varphi)\right)_{k \in \mathbb{Z}}$ be the sequence of gap lengths,

$$
\gamma_{k}(\varphi):=\lambda_{k}^{+}(\varphi)-\lambda_{k}^{-}(\varphi)
$$

In Section 4 we prove the following

THEOREM 1.3. - For $\varphi \in L^{2}\left(S^{1} ; \mathbb{C}\right)$, the following assertions are equivalent:

(i) $\lambda_{k}^{ \pm}(\varphi)=-\lambda_{-k}^{\mp}(\varphi)$ for any $k \geq 0$;

(ii) $\gamma_{k}(\varphi)=\gamma_{-k}(\varphi)$ for any $k \geq 1$

\section{Symmetries and spectra}

2.1. Periodic spectrum. - The periodic spectrum of the Zakharov-Shabat operator $L(\varphi)$ is given by

$$
\begin{aligned}
\operatorname{spec}_{\text {per }} L(\varphi):=\left\{\lambda \in \mathbb{C} \mid \exists F \in H_{\text {loc }}^{1}\left(\mathbb{R} ; \mathbb{C}^{2}\right),\right. & F \not \equiv 0 \text { with } L(\varphi) F=\lambda F \\
& \text { and } F(x+2)=F(x), \forall x \in \mathbb{R}\} .
\end{aligned}
$$

By [4], $\operatorname{spec}_{\text {per }} L(\varphi)$ consists of a sequence of real numbers $\left(\lambda_{k}^{ \pm}(\varphi)\right)_{k \in \mathbb{Z}}$, which can be ordered in such a way that (for all $k \in \mathbb{Z}$ )

$$
\lambda_{k}^{-}(\varphi) \leq \lambda_{k}^{+}(\varphi)<\lambda_{k+1}^{-}(\varphi)
$$

and $\lambda_{k}^{ \pm}(\varphi) \sim k \pi$ for $|k|$ large. We have the following

Proposition 2.1. - Let $\varphi \in L^{2}\left(S^{1} ; \mathbb{C}\right)$. Then, for any $k \in \mathbb{Z}$,

(i) $\lambda_{k}^{ \pm}\left(\mathrm{e}^{i \alpha} \varphi\right)=\lambda_{k}^{ \pm}(\varphi), \quad \lambda_{k}^{ \pm}(\varphi)=\lambda_{k}^{ \pm}\left(T_{\tau} \varphi\right) \quad(\forall \alpha \in \mathbb{R}, \tau \in \mathbb{R})$;

(ii) $\lambda_{k}^{ \pm}(\check{\varphi})=-\lambda_{-k}^{\mp}(\varphi), \quad \lambda_{k}^{ \pm}(\bar{\varphi})=-\lambda_{-k}^{\mp}(\varphi)$.

TOME $130-2002-\mathrm{N}^{\mathrm{O}} 4$ 
Proof. - (i) For $\alpha \in \mathbb{R}$ arbitrary, define $V_{\alpha}=\left(\begin{array}{cc}\mathrm{e}^{-i \alpha / 2} & 0 \\ 0 & \mathrm{e}^{i \alpha / 2}\end{array}\right)$. One easily verifies that

$$
L\left(\mathrm{e}^{i \alpha} \varphi\right)=V_{\alpha}^{-1} L(\varphi) V_{\alpha}
$$

and $L\left(T_{\tau} \varphi\right)=T_{\tau} L(\varphi) T_{-\tau}$. Thus both, $L\left(\mathrm{e}^{i \alpha} \varphi\right)$ and $L\left(T_{\tau} \varphi\right)$, are unitarily equivalent to $L(\varphi)$ and the claimed statement follows. To prove (ii) notice that

$$
L(-\check{\varphi})=-W^{-1} L(\varphi) W
$$

where $W$ is the unitary operator defined by

$$
W\left(\begin{array}{c}
Y \\
Z
\end{array}\right):=\left(\begin{array}{c}
\check{Y} \\
\check{Z}
\end{array}\right), \quad \text { with } \quad\left(\begin{array}{c}
Y \\
Z
\end{array}\right) \in L_{\mathrm{loc}}^{2}\left(\mathbb{R} ; \mathbb{C}^{2}\right) .
$$

Thus

$$
\operatorname{spec}_{\text {per }} L(-\check{\varphi})=-\operatorname{spec}_{\text {per }} L(\varphi) .
$$

Combining (2.4) and (i) we obtain $\lambda_{k}^{ \pm}(\check{\varphi})=-\lambda_{-k}^{\mp}(\varphi)$ for all $k \in \mathbb{Z}$. Consider $\lambda \in \operatorname{spec}_{\text {per }} L(\varphi)$ and choose $F \in H_{\text {loc }}^{1}\left(\mathbb{R} ; \mathbb{C}^{2}\right)$, satisfying $F(x+2)=$ $F(x)$ for all $x \in \mathbb{R}$ and $L(\varphi) F=\lambda F$. As $\lambda$ is real, $L(-\bar{\varphi}) \bar{F}=-\lambda \bar{F}$ and thus $-\lambda \in \operatorname{spec}_{\text {per }} L(-\bar{\varphi})$. Combined with (i), this leads to $\lambda_{k}^{ \pm}(\bar{\varphi})=-\lambda_{-k}^{\mp}(\varphi)$ for all $k \in \mathbb{Z}$.

2.2. Dirichlet spectra and divisors. - To study properties of the Dirichlet spectra it is convenient to consider the AKNS operator $H(\varphi)$ instead of $L(\varphi)$. Let

$$
F_{j}(x, \lambda ; \varphi):=\left(\begin{array}{c}
Y_{j}(x, \lambda ; \varphi) \\
Z_{j}(x, \lambda ; \varphi)
\end{array}\right), \quad j=1,2,
$$

be the fundamental solutions of $H(\varphi)$, i.e. the solutions to $H F=\lambda F$ such that

$$
F_{1}(0, \lambda ; \varphi)=\left(\begin{array}{l}
1 \\
0
\end{array}\right), \quad F_{2}(0, \lambda ; \varphi)=\left(\begin{array}{l}
0 \\
1
\end{array}\right) .
$$

For each $x \in \mathbb{R}$ and $\varphi \in L^{2}\left(S^{1} ; \mathbb{C}\right), F_{1}(x, \lambda ; \varphi)$ and $F_{2}(x, \lambda ; \varphi)$ are entire functions of $\lambda$. The two Dirichlet spectra are defined as follows

$$
\begin{aligned}
& \operatorname{spec}_{\text {Dir }}^{+} L(\varphi)=\left\{\lambda \in \mathbb{C} \mid Z_{1}(1, \lambda ; \varphi)=0\right\}, \\
& \operatorname{spec}_{\text {Dir }}^{-} L(\varphi)=\left\{\lambda \in \mathbb{C} \mid Y_{2}(1, \lambda ; \varphi)=0\right\} .
\end{aligned}
$$

It is proved in [4] that $\operatorname{spec}_{\text {Dir }}^{+} L(\varphi)$, resp. $\operatorname{spec}_{\text {Dir }}^{-} L(\varphi)$, consists of simple, real eigenvalues $\left(\mu_{k}(\varphi)\right)_{k \in \mathbb{Z}}$, resp. $\left(\nu_{k}(\varphi)\right)_{k \in \mathbb{Z}}$. The numerotation is chosen in such a way that $\left(\mu_{k}(\varphi)\right)_{k \in \mathbb{Z}}$ and $\left(\nu_{k}(\varphi)\right)_{k \in \mathbb{Z}}$ are strictly increasing satisfying $\mu_{k}(\varphi) \sim k \pi$ and $\nu_{k}(\varphi) \sim k \pi$ for $|k|$ large. Further introduce the function $\delta(\lambda ; \varphi)$, defined by

$$
\delta(\lambda ; \varphi)=Z_{2}(1, \lambda ; \varphi)-Y_{1}(1, \lambda ; \varphi) .
$$

BULLETIN DE LA SOCIÉTÉ MATHÉMATIQUE DE FRANCE 
Notice that for any $k \in \mathbb{Z}$,

$$
\delta\left(\mu_{k}(\varphi) ; \varphi\right)^{2}=\Delta\left(\mu_{k}(\varphi) ; \varphi\right)^{2}-4
$$

where

$$
\Delta(\lambda ; \varphi)=Y_{1}(1, \lambda ; \varphi)+Z_{2}(1, \lambda ; \varphi)
$$

is the discriminant.

Proposition 2.2. - Let $\varphi \in L^{2}\left(S^{1} ; \mathbb{C}\right)$. Then

(i) $\mu_{k}(-\varphi)=\nu_{k}(\varphi), \quad \nu_{k}(-\varphi)=\mu_{k}(\varphi)$ for all $k \in \mathbb{Z}$;

(ii) $\delta(\lambda ;-\varphi)=-\delta(\lambda ; \varphi)$.

REMARK. - For $\alpha \not \equiv 0, \pi(\bmod 2 \pi), \mu_{k}\left(\mathrm{e}^{i \alpha} \varphi\right)$ satisfies an equation involving $\operatorname{spec}_{\text {Dir }}^{ \pm} L(\varphi)$, the solution of which does not seem to be given in form of a closed expression.

Proof. - For $F=\left(\begin{array}{c}Y \\ Z\end{array}\right) \in H_{\text {loc }}^{1}\left(\mathbb{R} ; \mathbb{R}^{2}\right)$ define

$$
F^{\perp}=\left(\begin{array}{r}
Z \\
-Y
\end{array}\right)
$$

If $H(\varphi) F=\lambda F$ we have

$$
H(-\varphi) F^{\perp}=\lambda F^{\perp}
$$

Therefore

$$
F_{1}(x, \lambda ;-\varphi)=F_{2}^{\perp}(x, \lambda ; \varphi)
$$

and

$$
F_{2}(x, \lambda ;-\varphi)=-F_{1}^{\perp}(x, \lambda ; \varphi) .
$$

Using (2.11)-(2.12) one easily gets (i) and (ii).

Proposition 2.3. - For $\varphi \in L^{2}\left(S^{1} ; \mathbb{C}\right)$ and $k \in \mathbb{Z}$,

(i) $\mu_{k}(\bar{\varphi})=-\nu_{-k}(\varphi)$ and $\nu_{k}(\bar{\varphi})=-\mu_{-k}(\varphi)$;

(ii) $\delta\left(\mu_{k}(\bar{\varphi}), \bar{\varphi}\right)=-\delta\left(\nu_{-k}(\varphi), \varphi\right)$.

Proof. - (i) For $F=\left(\begin{array}{l}Y \\ Z\end{array}\right) \in H_{\text {loc }}^{1}\left(\mathbb{R} ; \mathbb{R}^{2}\right)$ define

$$
\widetilde{F}=\left(\begin{array}{c}
Z \\
Y
\end{array}\right) .
$$

If $H(\varphi) F=\lambda F, \widetilde{F}$ satisfies $H(\bar{\varphi}) \widetilde{F}=-\lambda \widetilde{F}$. Therefore

$$
F_{1}(x, \lambda ; \bar{\varphi})=\widetilde{F}_{2}(x,-\lambda ; \varphi), \quad F_{2}(x, \lambda ; \bar{\varphi})=\widetilde{F}_{1}(x,-\lambda ; \varphi) .
$$

Using (2.14) and the asymptotics of $\mu_{k}$ and $\nu_{k}$, one easily obtains (i). 
(ii) From (2.14) one deduces $\delta\left(\mu_{k}(\bar{\varphi}) ; \bar{\varphi}\right)=-\delta\left(-\mu_{k}(\bar{\varphi}) ; \varphi\right)$ and thus (ii) follows using (i).

Proposition 2.4. - For $\varphi \in L^{2}\left(S^{1} ; \mathbb{C}\right)$ and $k \in \mathbb{Z}$,

(i) $\mu_{k}(\check{\varphi})=-\nu_{-k}(\varphi)$ and $\nu_{k}(\check{\varphi})=-\mu_{-k}(\varphi)$;

(ii) $\delta\left(\mu_{k}(\check{\varphi}), \check{\varphi}\right)=\delta\left(\nu_{-k}(\varphi), \varphi\right)$.

REMARK. - By Proposition 2.1, 2.3 and 2.4, $\check{\varphi}$ and $\bar{\varphi}$ have the same periodic and the same Dirichlet spectra. They are only distinguished by

$$
\delta\left(\mu_{k}(\check{\varphi}), \check{\varphi}\right)=-\delta\left(\mu_{k}(\bar{\varphi}), \bar{\varphi}\right) \quad \forall k \in \mathbb{Z}
$$

Proof. — For $F=\left(\begin{array}{c}Y \\ Z\end{array}\right) \in H_{\text {loc }}^{1}\left(\mathbb{R} ; \mathbb{R}^{2}\right)$ we define

$$
F^{*}(x)=F(1-x) .
$$

If $H(\varphi) F=\lambda F$, then $F^{*}$ satisfies

$$
H(-\check{\varphi}) F^{*}=-\lambda F^{*} .
$$

By the definition of $\mu_{k}(\varphi)$, for any given $k \in \mathbb{Z}$,

$$
F_{1}\left(1, \mu_{k}(\varphi) ; \varphi\right)=Y_{1}\left(1, \mu_{k}(\varphi) ; \varphi\right)\left(\begin{array}{l}
1 \\
0
\end{array}\right)
$$

Therefore

$$
F_{1}\left(x,-\mu_{k}(\varphi) ;-\check{\varphi}\right)=\frac{1}{Y_{1}\left(1, \mu_{k}(\varphi) ; \varphi\right)} F_{1}\left(1-x, \mu_{k}(\varphi) ; \varphi\right) .
$$

Evaluated at $x=1,(2.17)$ leads to $Z_{1}\left(1,-\mu_{k}(\varphi) ;-\check{\varphi}\right)=0$. In view of the asymptotics of $\mu_{k}$ we conclude that $\mu_{k}(-\check{\varphi})=-\mu_{-k}(\varphi)$. Statement (i) then follows from Proposition 2.2 (i).

From (2.17) and (i), we deduce that

$$
Y_{1}\left(1, \mu_{-k}(-\check{\varphi}) ;-\check{\varphi}\right)=\frac{1}{Y_{1}\left(1, \mu_{k}(\varphi) ; \varphi\right)} .
$$

Further, the Wronskian identity (see [4])

$$
Y_{1}(1, \lambda ; \varphi) \cdot Z_{2}(1, \lambda ; \varphi)-Y_{2}(1, \lambda ; \varphi) \cdot Z_{1}(1, \lambda ; \varphi)=1
$$

implies that

$$
Z_{2}\left(1, \mu_{k}(\varphi) ; \varphi\right)=\frac{1}{Y_{1}\left(1, \mu_{k}(\varphi) ; \varphi\right)}
$$

Hence (2.18) and (2.20) yield

$$
\delta\left(\mu_{-k}(-\check{\varphi}) ;-\check{\varphi}\right)=-\delta\left(\mu_{k}(\varphi) ; \varphi\right) .
$$

Statement (ii) then follows by combining (2.21) and Proposition 2.2. 


\section{Symmetries in action-angle coordinates}

3.1. Actions. - Recall from [8] (see also [7]) that, for $\varphi \in L^{2}\left(S^{1} ; \mathbb{C}\right.$ ), the actions, $I_{k}(\varphi), k \in \mathbb{Z}$, are defined by

$$
I_{k}(\varphi):=\frac{2}{\pi} \int_{\lambda_{k}^{-}(\varphi)}^{\lambda_{k}^{+}(\varphi)}(-1)^{k+1} \frac{\mu \dot{\Delta}(\mu ; \varphi)}{\left|\Delta^{2}(\mu ; \varphi)-4\right|^{1 / 2}} \mathrm{~d} \mu
$$

where $\Delta(\mu ; \varphi)$ is the discriminant given by

$$
\Delta(\mu ; \varphi)=Y_{1}(1, \mu ; \varphi)+Z_{2}(1, \mu ; \varphi) .
$$

Proposition 3.1. - For $\varphi \in L^{2}\left(S^{1} ; \mathbb{C}\right)$ and $k \in \mathbb{Z}$

(i) $I_{k}\left(\mathrm{e}^{i \alpha} \varphi\right)=I_{k}(\varphi)$, for all $\alpha \in \mathbb{R}$;

(ii) $I_{k}(\bar{\varphi})=I_{-k}(\varphi)$;

(iii) $I_{k}(\check{\varphi})=I_{-k}(\varphi)$;

(iv) $I_{k}\left(T_{\tau} \varphi\right)=I_{k}(\varphi)$, for all $\tau \in \mathbb{R}$.

Proof. - Recall from [4] (cf. also [7]) that $\Delta^{2}(\mu ; \varphi)-4$ has a representation as an infinite product

$$
\begin{aligned}
\Delta^{2}(\mu ; \varphi)-4=-4\left(\lambda_{0}^{+}(\varphi)-\right. & \mu)\left(\lambda_{0}^{-}(\varphi)-\mu\right) \\
& \times \prod_{k \in \mathbb{Z}^{*}} \frac{\left(\lambda_{k}^{+}(\varphi)-\mu\right)\left(\lambda_{k}^{-}(\varphi)-\mu\right)}{k^{2} \pi^{2}}
\end{aligned}
$$

where the above infinite product $\prod_{k \in \mathbb{Z}^{*}} a_{k}$ is computed as the limit of the sequence $\left(\prod_{k=1}^{N} a_{k} a_{-k}\right)_{N \geq 1}$. Furthermore, for any $\mu$ in the open interval $\left(\lambda_{k}^{-}(\varphi), \lambda_{k}^{+}(\varphi)\right)$, sign $\Delta(\mu ; \varphi)=(-1)^{k}$. Therefore $\Delta(. ; \varphi)$, and thus $\left(I_{k}(\varphi)\right)_{k \in \mathbb{Z}}$, are uniquely determined by $\operatorname{spec}_{\text {per }} L(\varphi)$. In particular, statements (i) and (iv) follow from Proposition 2.1. To prove (ii) and (iii) notice that, by Proposition 2.1, for $\psi \in\{\bar{\varphi}, \breve{\varphi}\}$, $\operatorname{spec}_{\text {per }} L(\psi)=-\operatorname{spec}_{\text {per }} L(\varphi)$. Therefore $\Delta(\lambda ; \psi)=\Delta(-\lambda ; \varphi)$ and thus, by (3.1), one easily obtains for any $k \in \mathbb{Z}, I_{k}(\psi)=I_{-k}(\varphi)$.

3.2. Angles. - Denote by $\Sigma_{\varphi}$ the hyperelliptic Riemann surface,

$$
y=\sqrt{\Delta(\lambda ; \varphi)^{2}-4},
$$

and let $\beta_{j}=\beta_{j}(\varphi)(\forall j \in \mathbb{Z})$ be Abelian differential 1-forms of the third kind on $\Sigma_{\varphi}$, uniquely determined by the normalization conditions (see [7], [8]),

$$
\int_{a_{k}} \beta_{j}=2 \pi \delta_{k j}
$$

where for any $k \in \mathbb{Z}$ the cycle $a_{k} \equiv a_{k}(\varphi)$ is a contour around $\left[\lambda_{k}^{-}, \lambda_{k}^{+}\right]$with counterclockwise orientation on the sheet of the Riemann surface $\Sigma_{\varphi}$ determined by $i \sqrt{\Delta(\lambda ; \varphi)^{2}-4}>0$ for $\lambda_{0}^{+}<\lambda<\lambda_{1}^{-}$. For $k \in \mathbb{Z}$ with $I_{k}(\varphi) \neq 0$,

TOME $130-2002-\mathrm{N}^{\mathrm{O}} 4$ 
the angle $\theta_{k}(\varphi)$ is given by

$$
\theta_{k}(\varphi)=\sum_{j \in \mathbb{Z}} \int_{\lambda_{j}^{-}(\varphi)}^{\mu_{j}^{*}(\varphi)} \beta_{k}(\varphi)
$$

where $\mu_{j}^{*}(\varphi)$ denotes the point $\left(\mu_{j}(\varphi), \delta\left(\mu_{j}(\varphi) ; \varphi\right)\right)$ on $\Sigma_{\varphi}(c f .(2.8))$. As path of integration in (3.4) one chooses the straight line on the sheet of $\Sigma_{\varphi}$ containing $\mu_{j}^{*}(\varphi)$, connecting $\lambda_{j}^{-}$and $\mu_{j}^{*}(\varphi)$.

Proposition 3.2. - For $\varphi \in L^{2}\left(S^{1} ; \mathbb{C}\right), \alpha \in \mathbb{R}$, and $k \in \mathbb{Z}$ with $I_{k}(\varphi) \neq 0$,

$$
\theta_{k}\left(e^{i \alpha} \varphi\right) \equiv \theta_{k}(\varphi)+\alpha \quad(\bmod 2 \pi)
$$

Proof. - Denote by $R_{\alpha}$ the action of $M_{\alpha}$ expressed in Birkhoff coordinates, i.e.

$$
R_{\alpha}=\Phi^{-1} M_{\alpha} \Phi
$$

where $\Phi$ is the Birkhoff map, $\varphi=\Phi\left(\left(\sqrt{2 I_{j}} \mathrm{e}^{i \theta_{j}}\right)_{j \in \mathbb{Z}}\right)$. Notice that

$$
R_{\alpha+\beta}=\Phi^{-1} M_{\alpha} M_{\beta} \Phi=R_{\alpha} R_{\beta}, \quad R_{0}=\mathrm{Id},
$$

and

$$
\frac{\mathrm{d}}{\mathrm{d} \alpha} R_{\alpha}=\lim _{\beta \rightarrow 0} \frac{R_{\alpha+\beta}-R_{\alpha}}{\beta}=\lim _{\beta \rightarrow 0} \frac{R_{\beta}-\mathrm{Id}}{\beta} R_{\alpha}=i \cdot R_{\alpha} .
$$

Hence $R_{\alpha}=\mathrm{e}^{i \alpha} \mathrm{Id}$ and the claimed result follows.

Proposition 3.3. - For $\varphi \in L^{2}\left(S^{1} ; \mathbb{C}\right)$,

(i) $\theta_{k}(\check{\varphi}) \equiv \theta_{-k}(\varphi)(\bmod 2 \pi)$, for all $k \in \mathbb{Z}$ with $I_{-k}(\varphi) \neq 0$;

(ii) $\theta_{k}(\bar{\varphi}) \equiv-\theta_{-k}(\varphi)(\bmod 2 \pi)$, for all $k \in \mathbb{Z}$ with $I_{-k}(\varphi) \neq 0$.

Proof. - For $k \in \mathbb{Z}$ with $I_{k}(-\check{\varphi}) \neq 0$ we have, by (3.4) and Proposition 2.1,

$$
\theta_{k}(-\check{\varphi})=\sum_{j \in \mathbb{Z}} \int_{\lambda_{j}^{-}(-\check{\varphi})}^{\mu_{j}^{*}(-\check{\varphi})} \beta_{k}(-\check{\varphi})=\sum_{j \in \mathbb{Z}} \int_{-\lambda_{-j}^{+}(\varphi)}^{\mu_{j}^{*}(-\check{\varphi})} \beta_{k}(-\check{\varphi}) .
$$

To compute the latter integral, introduce the map $\sigma: \mathbb{C}^{2} \rightarrow \mathbb{C}^{2}$ defined by

$$
\sigma(\lambda, y)=(-\lambda,-y)
$$

Notice that for $(\lambda, y) \in \Sigma_{-\check{\varphi}}, \sigma(\lambda, y) \in \Sigma_{\varphi}$ as $\Delta^{2}(\lambda ;-\check{\varphi})=\Delta^{2}(-\lambda ; \varphi)$ (cf. formula (3.3) and Proposition 2.1), hence $\sigma: \Sigma_{-\check{\varphi}} \rightarrow \Sigma_{\varphi}$.

Changing coordinates according to $\sigma$, one has

$$
\int_{\sigma\left(a_{j}(-\check{\varphi})\right)} \sigma_{*} \beta_{k}(-\check{\varphi})=\int_{a_{j}(-\check{\varphi})} \beta_{k}(-\check{\varphi})=2 \pi \delta_{k j} .
$$

Furthermore, in view of Proposition 2.1, $\sigma\left(a_{j}(-\check{\varphi})\right)$ and $a_{-j}(\varphi)$ are homologous cycles, hence one deduces from the last identity that

$$
\int_{a_{-j}(\varphi)} \sigma_{*} \beta_{k}(-\check{\varphi})=2 \pi \delta_{j k}
$$

BULletin DE LA SOCIÉtÉ MATHÉMATIQUE DE FRANCE 
As $\beta_{k}(\varphi)$ is uniquely determined by its normalization conditions we conclude that

$$
\sigma_{*} \beta_{k}(-\check{\varphi})=\beta_{-k}(\varphi) .
$$

Further, using Proposition 2.2 and Proposition 2.4 we get

$$
\begin{aligned}
\sigma\left(\mu_{j}^{*}(-\check{\varphi})\right) & =\sigma\left(\mu_{j}(-\check{\varphi}), \delta\left(\mu_{j}(-\check{\varphi}) ;-\check{\varphi}\right)\right) \\
& =\sigma\left(-\mu_{-j}(\varphi),-\delta\left(\mu_{-j}(\varphi) ; \varphi\right)\right) \\
& =\left(\mu_{-j}(\varphi), \delta\left(\mu_{-j}(\varphi) ; \varphi\right)\right)=\mu_{-j}^{*}(\varphi) .
\end{aligned}
$$

Therefore, by a change of variable of integration in (3.5) we obtain

$$
\theta_{k}(-\check{\varphi})=\sum_{j \in \mathbb{Z}} \int_{\lambda_{-j}^{+}(\varphi)}^{\mu_{-j}^{*}(\varphi)} \beta_{k}(\varphi) .
$$

By contour integration and the normalization conditions $\int_{a_{j}} \beta_{k}=2 \pi \delta_{j k}$, we get for any $k \in \mathbb{Z}$,

$$
\int_{\lambda_{j}^{-}}^{\lambda_{j}^{+}} \beta_{k} \equiv \pi \delta_{j k} \quad(\bmod 2 \pi) .
$$

Therefore combining (3.4), (3.7) and (3.8) we obtain

$$
\theta_{k}(-\check{\varphi}) \equiv \theta_{-k}(\varphi)+\pi \quad(\bmod 2 \pi)
$$

which leads to (i), using Proposition 3.2 with $\alpha=\pi$.

In order to prove (ii), recall from Proposition 2.1 that $\lambda_{j}^{ \pm}(\check{\varphi})=\lambda_{j}^{ \pm}(\bar{\varphi})$. Hence $\Sigma_{\check{\varphi}}=\Sigma_{\bar{\varphi}}$ and $\beta_{k}(\check{\varphi})=\beta_{k}(\bar{\varphi})$ for any $k \in \mathbb{Z}$. Further, by Proposition 2.3 and 2.4,

$$
\mu_{j}(\check{\varphi})=\mu_{j}(\bar{\varphi}), \quad \delta\left(\mu_{j}(\check{\varphi}) ; \check{\varphi}\right)=-\delta\left(\mu_{j}(\bar{\varphi}) ; \bar{\varphi}\right) .
$$

From (3.4) it thus follows that

$$
\theta_{k}(\bar{\varphi})=\sum_{j \in \mathbb{Z}} \int_{\lambda_{j}^{-}(\check{\varphi})}^{f\left(\mu_{j}^{*}(\check{\varphi})\right)} \beta_{k}(\check{\varphi})
$$

where $f(\lambda, \delta)=(\lambda,-\delta)$ is the involution on $\Sigma_{\check{\varphi}}$ exchanging the two sheets. Hence $\beta_{k}(\varphi)$ satisfies

$$
\int_{f\left(a_{j}(\varphi)\right)} \beta_{k}(\varphi)=-2 \pi \delta_{k j} .
$$

By changing coordinates according to $f$ one then gets

$$
\int_{a_{j}(\varphi)} f^{*} \beta_{k}(\varphi)=-2 \pi \delta_{k j}
$$

TOME $130-2002-\mathrm{N}^{\mathrm{O}} 4$ 
and hence $f^{*} \beta_{k}(\varphi)=-\beta_{k}(\varphi)$ for any $k \in \mathbb{Z}$. Therefore, by a change of variable of integration in (3.9), we get

$$
\theta_{k}(\bar{\varphi})=-\sum_{j \in \mathbb{Z}} \int_{\lambda_{j}^{-}(\check{\varphi})}^{\mu_{j}^{*}(\check{\varphi})} \beta_{k}(\check{\varphi}) \equiv-\theta_{k}(\check{\varphi}) .
$$

Combining (3.10) and (i) we obtain (ii).

Proposition 3.4. - Let $\varphi \in L^{2}\left(S^{1} ; \mathbb{C}\right), \tau \in \mathbb{R}$ and $k \in \mathbb{Z}$ with $I_{k}(\varphi) \neq 0$. Then

$$
\theta_{k}\left(T_{\tau} \varphi\right)=\theta_{k}(\varphi)+2 \pi k \tau
$$

Proof. — By continuity, it suffices to consider $\varphi \in H^{1}\left(S^{1} ; \mathbb{C}\right)$. The translation flow $T_{\tau} \varphi(\cdot)=\varphi(\tau+\cdot)$ is the Hamiltonian flow associated with the Hamiltonian (see [2] or [4]).

$$
\mathcal{H}_{1}(\varphi)=\frac{i}{2} \int_{S^{1}}\left(\bar{\varphi} \varphi^{\prime}-\varphi \bar{\varphi}^{\prime}\right) \mathrm{d} x
$$

which commutes with the NLS-Hamiltonian. Thus for $\varphi=\Phi\left(\left(\sqrt{2 I_{j}} \mathrm{e}^{i \theta_{j}}\right)_{j \in \mathbb{Z}}\right)$ and $k \in \mathbb{Z}$ with $I_{k} \neq 0$

$$
\theta_{k}\left(T_{\tau} \varphi\right)=\theta_{k}(\varphi)+w_{k}(I) \tau
$$

where

$$
w_{k}(I):=\frac{\partial \mathcal{H}_{1}}{\partial I_{k}}(I)
$$

is the $k$-th frequency of the translation flow. Since $\theta_{k}\left(T_{1} \varphi\right)=\theta_{k}(\varphi)$, there exists for any $I \in \ell^{1}\left(\mathbb{Z} ; \mathbb{R}_{\geq 0}\right)$ with $I_{k}>0$, an integer $n_{k}(I) \in \mathbb{Z}$ such that $w_{k}(I)=2 \pi n_{k}(I)$. Furthermore, since $w_{k}(I)$ is continuous and $n_{k}(I)$ takes discrete values, $n_{k}(I)$ does not depend on $I$, i.e. $n_{k}(I) \equiv n_{k}$. From Lemma 3.5 below we deduce that for a 1-gap potential $\varphi$ with $I_{j}(\varphi)=0$ for all $j \neq k$ and $I_{k}(\varphi)>0$ the frequency $w_{k}(I)$ is given by $w_{k}(I)=2 \pi k$.

REMARK. - We note that the identities $\partial \mathcal{H}_{1} / \partial I_{k}=2 \pi k$ (for all $k \in \mathbb{Z}$ ) established in the proof above together with $\mathcal{H}_{1}(0)=0$ implies that the following trace formula holds for any $\varphi \in H^{1}\left(S^{1} ; \mathbb{C}\right)$

$$
\mathcal{H}_{1}(\varphi)=\sum_{k \in \mathbb{Z}} 2 k \pi I_{k}
$$

LemMA 3.5. - For $k \in \mathbb{Z}$,

$$
\left\{\varphi \in L^{2}\left(S^{1} ; \mathbb{C}\right) \mid \gamma_{j}(\varphi)=\gamma \delta_{k j}, j \in \mathbb{Z}\right\}=\left\{\varphi(x)=c \mathrm{e}^{2 i \pi k x}|c \in \mathbb{C},| c \mid=\frac{1}{2} \gamma\right\} .
$$

Proof. - A straightforward computation proves that for $\varphi \in L^{2}\left(S^{1} ; \mathbb{C}\right)$ and $j, k \in \mathbb{Z}$ arbitrary,

$$
\gamma_{j}\left(\mathrm{e}^{-2 i \pi k x} \varphi\right)=\gamma_{j-k}(\varphi)
$$

BULletin DE LA SOCIÉtÉ MATHÉMATIQUE DE FRANCE 
Further one knows (cf. [4])

(3.15) $\left\{\varphi \in L^{2}\left(S^{1} ; \mathbb{C}\right) \mid \gamma_{j}(\varphi)=\gamma \delta_{0 j}, \forall j \in \mathbb{Z}\right\}=\left\{\varphi(x)=c|c \in \mathbb{C}| c \mid,=\frac{1}{2} \gamma\right\}$.

Combining (3.14) and (3.15), Lemma 3.5 follows.

\section{Applications}

4.1. Symmetries of the Hamiltonian and its frequencies. - As already mentioned in the introduction, the NLS Hamiltionian $\mathcal{H}$ is invariant under $\mathcal{S}_{2}$. When $\mathcal{H}$ is expressed with respect to action variables, $\mathcal{H}=\mathcal{H}(I)$, this invariance of $\mathcal{H}$ leads to our first application. For $I=\left(I_{k}\right)_{k \in \mathcal{Z}}$, denote by $\mathcal{J}(I)$ the sequence given by

$$
\mathcal{J}(I)_{k}:=I_{-k} \quad \forall k \in \mathbb{Z} .
$$

Denote the $j$-th frequency by

$$
\omega_{j}:=\frac{\partial \mathcal{H}}{\partial I_{j}}
$$

Proposition 4.1. - (i) $\mathcal{H}(\mathcal{J}(I))=\mathcal{H}(I)$.

(ii) For any $I=\left(I_{k}\right)_{k \in \mathbb{Z}}$ with $\mathcal{J}(I)=I$ it follows that $\omega_{j}(I)=\omega_{-j}(I)$ for any $j \in \mathbb{Z}$.

Proof. - (i) follows from combining the two identities $\mathcal{H}(\check{\varphi})=\mathcal{H}(\varphi)$ and $I(\check{\varphi})=\mathcal{J}(I(\varphi))$ (Proposition 3.1).

(ii) Write $\mathcal{H}$ as a function of $r_{k}:=\frac{1}{2}\left(I_{k}+I_{-k}\right)(k \geq 0)$ and $\rho_{k}:=\frac{1}{2}\left(I_{k}-I_{-k}\right)$ $(k \geq 1)$. By (i), $\partial \mathcal{H} / \partial \rho_{k}=0$ at points where $\mathcal{J}(I)=I$.

4.2. Symmetric phase spaces. - For $\alpha \in \mathbb{R}$, introduce the subspace

$$
P_{\alpha}:=\left\{\varphi \in L^{2}\left(S^{1} ; \mathbb{C}\right) \mid \mathrm{e}^{i \alpha} \check{\varphi}=\varphi\right\} .
$$

Notice that for $\alpha=\pi$, respectively $\alpha=0, P_{\alpha} \cap C^{\infty}$ is the phase space consisting of elements $\varphi \in C^{\infty}$ satisfying a generalized Dirichlet respectively Neumann condition, i.e. for all $k \geq 0$,

$$
\begin{aligned}
& \partial_{x}^{2 k} \varphi(0)=\partial_{x}^{2 k} \varphi(1)=0 \quad \text { (Dirichlet), } \\
& \partial_{x}^{2 k+1} \varphi(0)=\partial_{x}^{2 k+1} \varphi(1)=0 \quad \text { (Neumann). }
\end{aligned}
$$

Next, introduce the subspace

$$
Q_{\alpha}:=\left\{\varphi \in L^{2}\left(S^{1} ; \mathbb{C}\right) \mid \mathrm{e}^{i \alpha} \bar{\varphi}=\varphi\right\} .
$$

Notice that $\mathrm{e}^{i \alpha} \bar{\varphi}=\varphi$ means that $\varphi$ is of the form $\varphi=\mathrm{e}^{i \alpha / 2} f(x)$ with $f(x)$ a real valued function.

Proposition 4.2 provides a charaterization of $P_{\alpha}$ and $Q_{\alpha}$ in terms of actionangle variables. Recall that $\Phi$ denotes the Birkhoff map,

$$
\varphi=\Phi\left(\left(\sqrt{2 I_{k}} \mathrm{e}^{i \theta_{k}}\right)_{k \in \mathbb{Z}}\right) .
$$

TOME $130-2002-\mathrm{N}^{\mathrm{O}} 4$ 
Proposition 4.2. - The following statements hold:

$$
\begin{aligned}
P_{0}=\left\{\Phi\left(\left(\sqrt{2 I_{k}} \mathrm{e}^{i \theta_{k}}\right)_{k \in \mathbb{Z}}\right) \mid I_{k}=I_{-k}, \forall k \geq 1,\right. \\
\left.\theta_{k} \equiv \theta_{-k}(\bmod 2 \pi), \forall k \geq 1 \text { with } I_{k} \neq 0\right\}
\end{aligned}
$$

(ii) For $\alpha \neq \equiv 0(\bmod 2 \pi)$,

$$
\begin{array}{r}
P_{\alpha}=\left\{\Phi\left(\left(\sqrt{2 I_{k}} \mathrm{e}^{i \theta_{k}}\right)_{k \in \mathbb{Z}}\right) \mid I_{0}=0, I_{k}=I_{-k}, \forall k \geq 1\right. \\
\left.\theta_{k} \equiv \theta_{-k}+\alpha(\bmod 2 \pi), \forall k \geq 1 \text { with } I_{k} \neq 0\right\} ;
\end{array}
$$

(iii) $Q_{\alpha}=\left\{\Phi\left(\left(\sqrt{2 I_{k}} \mathrm{e}^{i \theta_{k}}\right)_{k \in \mathbb{Z}}\right) \mid I_{k}=I_{-k}, \forall k \geq 1\right.$,

$$
\left.\theta_{k} \equiv-\theta_{-k}+\alpha(\bmod 2 \pi), \forall k \geq 0 \text { with } I_{k} \neq 0\right\}
$$

Proof. - (i) Let us denote by $\widetilde{P}_{0}$ the set on the right side of equality (i). If $\varphi \in P_{0}$, Proposition 3.1 and 3.3 imply $\varphi \in \widetilde{P}_{0}$. Conversely, if $\varphi \in \widetilde{P}_{0}$ then by Proposition 3.1, $3.3 I_{k}(\check{\varphi})=I_{k}(\varphi)$ for any $k \in \mathbb{Z}$ and $\theta_{k}(\check{\varphi})=\theta_{k}(\varphi)$ for any $k \in \mathbb{Z}$ with $I_{k} \neq 0$. Thus $\check{\varphi}=\varphi$ since $\Phi$ is one to one.

(ii) Let us denote by $\widetilde{P}_{\alpha}$ the set on the right side of the equality (ii). If $\varphi \in \widetilde{P}_{\alpha}$ then, by Proposition $3.1,3.2,3.3, I_{k}\left(\mathrm{e}^{i \alpha} \check{\varphi}\right)=I_{k}(\varphi)$ for any $k \in \mathbb{Z}$ and $\theta_{k}\left(\mathrm{e}^{i \alpha} \check{\varphi}\right)=\theta_{k}(\varphi)$ for any $k \in \mathbb{Z}$ with $I_{k} \neq 0$, hence $\varphi \in P_{\alpha}$. Conversely, if $\varphi \in P_{\alpha}$ we have $I_{k}(\varphi)=I_{-k}(\varphi)$ for any $k \in \mathbb{Z}$ and $\theta_{k}(\varphi) \equiv \theta_{-k}(\varphi)+\alpha(\bmod 2 \pi)$ for any $k \in \mathbb{Z}$ with $I_{k}(\varphi) \neq 0$. In particular, if $I_{0}(\varphi) \neq 0, \theta_{0}(\varphi) \equiv \theta_{0}(\varphi)+$ $\alpha(\bmod 2 \pi)$. As $\alpha \neq \equiv 0(\bmod 2 \pi)$, it follows that $I_{0}(\varphi)=0$ and thus $\varphi \in \widetilde{P}_{\alpha}$.

The statement (iii) is proved in a similar way.

Further, introduce the subspace

$$
E_{\alpha}:=\left\{\varphi \in L^{2}\left(S^{1} ; \mathbb{C}\right) \mid \mathrm{e}^{i \alpha} \check{\varphi}=\bar{\varphi}\right\}
$$

and let $E:=E_{0}$. Notice that $E$ is also given by

$$
E=\left\{\varphi \in L^{2}\left(S^{1} ; \mathbb{C}\right) \mid \operatorname{Re} \varphi \text { is even, } \operatorname{Im} \varphi \text { is odd }\right\}
$$

and recall from [4] that

$$
\begin{aligned}
E & =\left\{\varphi \in L^{2}\left(S^{1} ; \mathbb{C}\right) \mid \operatorname{spec}_{\text {per }} L(\varphi)=\operatorname{spec}_{\text {Dir }}^{+} L(\varphi) \cup \operatorname{spec}_{\text {Dir }}^{-} L(\varphi)\right\} \\
& =\left\{\varphi \in L^{2}\left(S^{1} ; \mathbb{C}\right) \mid \forall k \in \mathbb{Z}, \mu_{k}(\varphi) \in\left\{\lambda_{k}^{+}(\varphi), \lambda_{k}^{-}(\varphi)\right\}\right\}
\end{aligned}
$$

Proposition 4.3. - One has

$$
E_{\alpha}=\left\{\Phi\left(\left(\sqrt{2 I_{k}} \mathrm{e}^{i \theta_{k}}\right)_{k \in \mathbb{Z}}\right) \mid \theta_{k} \equiv \frac{1}{2} \alpha(\bmod \pi), \forall k \in \mathbb{Z} \text { with } I_{k} \neq 0\right\} .
$$

Proof. - The statement is proved in a similar way as Proposition 4.2. 
Remark. - In particular for $\alpha=0$, Proposition 4.3, combined with (4.3), shows that

$$
\begin{aligned}
& \left\{\varphi \in L^{2}\left(S^{1} ; \mathbb{C}\right) \mid \operatorname{spec}_{\text {per }} L(\varphi)=\operatorname{spec}_{\text {Dir }}^{+} L(\varphi) \cup \operatorname{spec}_{\text {Dir }}^{-} L(\varphi)\right\} \\
& \quad=\left\{\Phi\left(\left(\sqrt{2 I_{k}} \mathrm{e}^{i \theta_{k}}\right)_{k \in \mathbb{Z}}\right) \mid \theta_{k} \equiv 0(\bmod \pi), \forall k \in \mathbb{Z} \text { with } I_{k} \neq 0\right\} .
\end{aligned}
$$

4.3. Symmetric spectrum. - In this subsection we present two spectral results.

Recall from [4] that the sequence of the gap lengths,

$$
\left(\gamma_{k}(\varphi)\right)_{k \in \mathbb{Z}}=\left(\lambda_{k}^{+}(\varphi)-\lambda_{k}^{-}(\varphi)\right)_{k \in \mathbb{Z}},
$$

uniquely determines the periodic spectrum of $L(\varphi)$. Similarly, the sequence of actions $\left(I_{k}(\varphi)\right)_{k \in \mathbb{Z}}$ determines uniquely the periodic spectrum of $L(\varphi)$.

Proposition 4.4. - For any $\varphi, \psi \in L^{2}\left(S^{1} ; \mathbb{C}\right)$ the following statements are equivalent:

(i) $\operatorname{spec}_{\text {per }} L(\varphi)=\operatorname{spec}_{\text {per }} L(\psi)$;

(ii) $I_{k}(\varphi)=I_{k}(\psi)$, for all $k \in \mathbb{Z}$.

Before proving Proposition 4.4, we recall the following result from [4] (see also [3] or [7])

Lemma 4.5. - For any $\varphi_{0} \in L^{2}\left(S^{1} ; \mathbb{C}\right)$ there exists $\varphi \in L^{2}\left(S^{1} ; \mathbb{C}\right)$ such that

(i) $\operatorname{spec}_{\text {per }} L(\varphi)=\operatorname{spec}_{\text {per }} L\left(\varphi_{0}\right)$;

(i) $\mu_{k}(\varphi)=\lambda_{k}^{-}(\varphi)\left(=\lambda_{k}^{-}\left(\varphi_{0}\right)\right)$, for all $k \in \mathbb{Z}$.

Proof of Proposition 4.4. - As the actions are defined in terms of spectral data, statement (i) implies (ii). Conversely, assume that $I_{k}(\varphi)=I_{k}(\psi)$ for any $k \in \mathbb{Z}$. According to Lemma 4.5 , there exists a potential $\varphi_{0}$ in $L^{2}\left(S^{1} ; \mathbb{C}\right)$

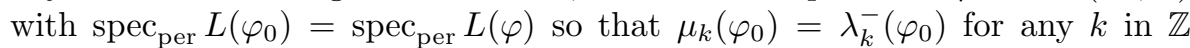
and hence, by the definition of $\theta_{k}$,

$$
\theta_{k}\left(\varphi_{0}\right) \equiv 0 \quad(\bmod 2 \pi) \text { for any } k \in \mathbb{Z} \text { with } I_{k}\left(\varphi_{0}\right) \neq 0 .
$$

Similarly there exists $\psi_{0}$ in $L^{2}\left(S^{1} ; \mathbb{C}\right)$ with $\operatorname{spec}_{\text {per }} L\left(\psi_{0}\right)=\operatorname{spec}_{\text {per }} L(\psi)$ and

$$
\theta_{k}\left(\psi_{0}\right) \equiv 0 \quad(\bmod 2 \pi) \text { for any } k \in \mathbb{Z} \text { with } I_{k}\left(\psi_{0}\right) \neq 0 .
$$

As (i) implies (ii) one knows that $I_{k}\left(\varphi_{0}\right)=I_{k}(\varphi)$ and $I_{k}\left(\psi_{0}\right)=I_{k}(\psi)$ for any $k \in \mathbb{Z}$. As $I_{k}(\varphi)=I_{k}(\psi)$ for any $k \in \mathbb{Z}$ by assumption it then follows $I_{k}\left(\varphi_{0}\right)=I_{k}\left(\psi_{0}\right)$ for any $k \in \mathbb{Z}$. Combined with (4.4)-(4.5) it then follows from the uniqueness of the Birkhoff map that $\varphi_{0}=\psi_{0}$. This implies that $\operatorname{spec}_{\text {per }} L(\varphi)=\operatorname{spec}_{\text {per }} L(\psi)$ as claimed.

In Theorem 4.1, and 4.2 below, we prove that the periodic spectrum is symmetric if and only if the sequence of the actions is symmetric (Theorem 4.1) or, if and only if the sequence of the gap lengths is symmetric (Theorem 4.2).

TOME $130-2002-\mathrm{N}^{\mathrm{O}} 4$ 
TheOREM 4.1. - For any $\varphi \in L^{2}\left(S^{1} ; \mathbb{C}\right)$, the following two statements are equivalent:

(i) $\lambda_{k}^{ \pm}(\varphi)=-\lambda_{-k}^{\mp}(\varphi)$, for all $k \in \mathbb{Z}$;

(ii) $I_{k}(\varphi)=I_{-k}(\varphi)$, for all $k \in \mathbb{Z}$.

Proof. - In view of the formula for the actions, (i) implies (ii). Conversely, assume (ii) holds. As the Birkhoff map is bijective, there exists $\varphi_{0} \in L^{2}\left(S^{1} ; \mathbb{C}\right)$ such that $I_{k}\left(\varphi_{0}\right)=I_{k}(\varphi)$, for all $k \in \mathbb{Z}$, and $\theta_{k}\left(\varphi_{0}\right) \equiv \theta_{-k}\left(\varphi_{0}\right)(\bmod 2 \pi)$ for any $k \in \mathbb{Z}$ with $I_{k}\left(\varphi_{0}\right) \neq 0$. By Proposition 4.2 (i), it follows that $\check{\varphi}_{0}=\varphi_{0}$. By Proposition 2.1 (ii), this then implies that $\operatorname{spec}_{\text {per }} L\left(\varphi_{0}\right)$ is symmetric. Since $\varphi_{0}$ and $\varphi$ are isospectral by Proposition $4.4, \operatorname{spec}_{\text {per }} L(\varphi)$ is symmetric.

THEOREM 4.2. - For any $\varphi \in L^{2}\left(S^{1} ; \mathbb{C}\right)$, the following two statements are equivalent:

(i) $\lambda_{k}^{ \pm}(\varphi)=-\lambda_{-k}^{\mp}(\varphi)$, for all $k \in \mathbb{Z}$;

(ii) $\gamma_{k}(\varphi)=\gamma_{-k}(\varphi)$, for all $k \in \mathbb{Z}$.

Proof. — Clearly, (i) implies (ii). Conversely, assume that $\varphi \in L^{2}\left(S^{1} ; \mathbb{C}\right)$ satisfies $\gamma_{k}(\varphi)=\gamma_{-k}(\varphi)$ for any $k \in \mathbb{Z}$. By Lemma 4.5 there exists $\varphi_{0} \in L^{2}\left(S^{1} ; \mathbb{C}\right)$ with $\operatorname{spec}_{\text {per }} L\left(\varphi_{0}\right)=\operatorname{spec}_{\text {per }} L(\varphi)$ so that $\mu_{k}\left(\varphi_{0}\right)=\lambda_{k}^{-}\left(\varphi_{0}\right)$ for any $k \in \mathbb{Z}$. Hence $\theta_{k}\left(\varphi_{0}\right)=0$ for any $k \in \mathbb{Z}$ with $I_{k}\left(\varphi_{0}\right) \neq 0$. As $\gamma_{k}\left(\varphi_{0}\right)=\gamma_{-k}\left(\varphi_{0}\right)$ for any $k \in \mathbb{Z}$ we deduce from Proposition 2.1 (ii) that $\gamma_{k}\left(\varphi_{0}\right)=\gamma_{k}\left(\check{\varphi}_{0}\right)$ for any $k \in \mathbb{Z}$ and hence $\operatorname{spec}_{\text {per }} L\left(\varphi_{0}\right)=\operatorname{spec}_{\text {per }} L\left(\check{\varphi}_{0}\right)$ (cf. [4]). By Theorem 4.1 one concludes that $I_{k}\left(\varphi_{0}\right)=I_{k}\left(\check{\varphi}_{0}\right)$ for any $k \in \mathbb{Z}$. Apply Proposition 3.1 to conclude that $I_{k}(\varphi)=I_{-k}\left(\varphi_{0}\right)$ for any $k \in \mathbb{Z}$. Together with the identities $\theta_{k}\left(\varphi_{0}\right)=\theta_{-k}\left(\varphi_{0}\right)(=0)$ for any $k \in \mathbb{Z}$ with $I_{k}\left(\varphi_{0}\right) \neq 0$ it then follows from Proposition 4.2 (i) that $\varphi_{0} \in P_{0}$, i.e. $\varphi_{0}=\check{\varphi}_{0}$, and thus by Proposition 2.1 (ii), spec $_{\text {per }} L\left(\varphi_{0}\right)$ is symmetric. As $\operatorname{spec}_{\text {per }} L(\varphi)=\operatorname{spec}_{\text {per }} L\left(\varphi_{0}\right)$, the claimed result then follows.

4.4. Additional symmetries of the spectrum. - Theorem 4.2 suggests that the 0's gap plays a special role for symmetry properties of the spectrum. Indeed, if we consider the action $\rho$ of $\mathbb{Z}$ on $L^{2}\left(S^{1} ; \mathbb{C}\right)$ given by $\rho(j) \varphi(x)=$ $\mathrm{e}^{-2 \pi i j x} \varphi(x)$, one verifies easily $(c f .(3.14))$ that the following statements are equivalent

(i) $\lambda_{j+k}^{ \pm}(\varphi)=-\lambda_{j-k}^{\mp}(\varphi)+2 j \pi$, for all $k \in \mathbb{Z}$;

(ii) $\gamma_{j+k}(\varphi)=\gamma_{j-k}(\varphi)$, for all $k \in \mathbb{Z}$. 


\section{BIBLIOGRAPHY}

[1] Bourgain (J.) - Fourier transform restriction phenomena for certain lattice subsets and applications to nonlinear evolution equations, GAFA, t. 3 (1993), pp. 107-156.

[2] Faddeev (L.D.) \& Takhtajan (L.A.) - Hamiltonian methods in the theory of solitons, Springer, 1987.

[3] GrÉBert (B.) - Problèmes spectraux inverses pour les systèmes AKNS sur la droite réelle, Thèse, Université Paris-Nord, 1990.

[4] Grébert (B.) \& Guillot (J.C.) - Gaps of one dimensional periodic AKNS systems, Forum Math, t. 5 (1993), pp. 459-504.

[5] GréBert (B.) \& KAPpeler (T.) - Perturbations of the NLS equation, to appear in Milan J. of Math.

[6] _ Théorème de type KAM pour l'équation de Schrödinger non linéaire, C. R. Acad. Sci. Paris Sér. I Math., t. 327 (1998), pp. 473-478.

[7] Grébert (B.), Kappeler (T.) \& Pöschel (J.) - Normal form theory for $N L S$, preliminary version available (please contact authors).

[8] McKean (H.P.) \& VAninsky (K.L.) - Action-angle variables for the cubic Schrödinger equation, CPAM, t. 50 (1997), pp. 489-562. 